\title{
Frequency of Contrast Induced Nephropathy (CIN) Following Exposure to Contrast in Coronary Angiography, in Diabetics with Previously Normal Renal Function
}

\author{
Uzma Sajjad, ${ }^{1}$ Irtza Ali, ${ }^{2}$ Zubair Akram ${ }^{3}$
}

\begin{abstract}
Introduction: Contrast - induced nephropathy (CIN), is an acute kidney injury state, which has managed to catch the attention of cardiologists, as a result of increasing cardiac invasive interventions. Lately there has been a lot of research to get insight in the etiology, pathogenesis, recognition, diagnosis and prevention of CIN, so that the long term adverse effects can be avoided. Much of the research has been in the form of retrospective or observational studies on groups of patients undergoing angiographic contrast exposure.
\end{abstract}

Objective: The objective of the study was to:

Determine the frequency of CIN in diabetics undergoing coronary angiography.

Study Design: Case series.

Settings: Department of Cardiology, Jinnah Hospital, Lahore.

Duration of Study: Six months $\left(1^{\text {st }}\right.$ June, 2014 to $30^{\text {th }}$ November, 2014).

Conflict of Interest: No

Funding Source: No

Sajjad U. ${ }^{1}$

Senior Registrar

Department of Cardiology, Mayo Hospital, Lahore

Ali I. ${ }^{2}$

RMO, Post op Surgical Care, Spire Hospital, NES, UK

Akram Z. ${ }^{3}$

Professor and Head of Cardiology Department

AIMC / Jinnah Hospital, Lahore
Subjects and Methods: 155 patients fulfilling the selection criteria were included in the study. They were admitted through medical emergency and outdoor department and admitted in CCU, Jinnah Hospital Lahore. Written informed consent was taken. Patient related information (name, age, sex and address) was obtained. These patients underwent coronary angiography. Post angiogram, serum analysis was done daily during hospital stay of patient to assess serum creatinine. CIN was labeled as per operational definition and thus frequency of CIN in type I and type II diabetics undergoing angiography was determined by serial creatinine monitoring.

Results: The frequency of increase in serum createnine $\geq 0.5 \mathrm{mg} / \mathrm{dl}$ after PCI leading to CIN in diabetics undergoing coronary angiography was recorded in $7.10 \%(n=11)$ out of a total of 155 cases.

Conclusion: We concluded that the frequency of CIN is not very high in diabetic patients undergoing coronary angiography. However, it continues to be a clinically challenging adverse event in all patients, especially diabetics, having coronary angiography for diagnostic or therapeutic purposes. Therefore, these patients should be recognized and monitored carefully to avoid long-term adverse effects of CIN.

Keywords: Coronary artery disease, coronary angiography, diabetes, contrast induced nephropathy, frequency.

\section{Introduction}

Ischemic Heart Disease (IHD) poses an ever expanding health and economic burden in the present times, especially in the developing countries. Today, $30 \%$ of 
the total deaths are caused by IHD. Acute Myocardial Infarction (AMI) and its frightful complication spectrum is the most feared presentation of IHD, having substantial morbidity and mortality. Owing to the remarkable evolution in pharmacological and interventional therapies to treat AMI and its complications, it is now possible to save more lives and decrease cardiovascular morbidity. ${ }^{1,2}$ In the last few decades, the implementation of Coronary angiography for diagnostic as well as therapeutic purposes has increased massively. CIN is a poor prognostic factor with increased mortality. A lot more clinical data and studies are required to fully establish the relation between CIN and death due to CIN. CIN leads to prolonged hospital stay. CIN is the cause of nearly $10 \%$ of AKI that develop during hospital stay, causing it to be a major prognostic factor in increased mortality and morbidity. The mechanisms of development of CIN are not completely understood yet. ${ }^{3,4}$

Despite the observation that diabetic patients undergoing PCI are at an increased risk for developing CIN, no definite pharmacological therapy has been defined for preventive practice. Thus, the identification of patients who are at an enhanced risk of development of CIN is pertinent. ${ }^{5}$

One study reported the frequency of CIN in diabetics as only $5.2 \% .^{6}$ According to another study, the frequency of CIN was reported to be $18.4 \%$ in diabetics. ${ }^{7}$ While one study reported a very high rate i.e. CIN occurred in $35.7 \%$ of diabetic patients. ${ }^{8}$ Usually, CIN appears within $24-48$ hours of exposure to the contrast medium, serum creatinine levels increase achieving the maximum value in 3-5 days. In majority of the cases, the renal functions return to normal values or baseline in about $7-21$ days post contrast exposure. $^{9}$

The rationale of this study was to observe and find out the extent and frequency of CIN in diabetic patients who are undergoing coronary angiography. Literature has showed that CIN is more in diabetic patients after PCI but there is also controversy in results. Some studies have reported very high rate of post angiography CIN but some reported its incidence as very low. Through this study we wanted to confirm the frequency of post-angiography CIN, as there is no local data available regarding this complication.

\section{Methodology}

This was a descriptive study, conducted at Department of Cardiology, Jinnah Hospital Lahore, Pakistan for a duration of 6 months, from $1^{\text {st } J u n e ~} 2014$ to $31^{\text {st }}$ November 2014. CIN was defined as "a new/acute rise in the renal function tests i.e., rise in serum creatinine by $25 \%$ or greater than $0.5 \mathrm{mg} / \mathrm{dL}$ when compared with the baseline serum creatinine or an acute decline in the estimated glomerular filtration rate (eGFR) by more than $25 \%$, after exposure to angiographic contrast material .However, other causes of acute renal injury, structural renal disease or pre-existing renal failure are to be ruled out first". 9 Diabetes was labeled as Fasting plasma glucose level $>7 \mathrm{mmol} / \mathrm{l}(126 \mathrm{mg} / \mathrm{dl})$ and Random plasma glucose level $>11.1 \mathrm{mmol} / \mathrm{l}$ (200 $\mathrm{mg} / \mathrm{dl}$ ) or those patients who are already taking antidiabetic drugs.

The sampling technique was Non-probability, purposive sampling. A sample size of 155 cases was taken. The patients were selected from the outdoor and emergency cases presenting to Cardiology Department, Jinnah Hospital with coronary artery disease in whom coronary angiography was being planned.

The inclusion criteria included Diabetic patients of age $30-80$ years, of either gender, undergoing PCI after first episode of MI. Exclusion criteria was defined as patients with baseline serum creatinine $>1.3$ $\mathrm{mg} / \mathrm{dl}$ before PCI, patients with advanced liver disease, patients with previous history of PCI or coronary artery bypass grafting, structural kidney disease ruled out by ultrasonography (renal stones, hydronephrosis, obstructive uropathies, and polycystic disease) and previous history of alcohol use.

155 patients fulfilling the inclusion criteria were selected for the study through medical emergency and were admitted in CCU, Jinnah Hospital Lahore. Written informed consent was taken. Patient related information (name, age, sex and address) was obtained. Then patients underwent angiography under a single cardiologist. Blood sampling was done for the serum creatinine levels at the time of admission and again repeated before coronary angiography. Serum creatinine was again repeated 24 and 72 hours after the angiography.

Data analysis was done on SPSS version 17. Quantitative variables like age and post procedure serum creatinine value were presented by mean \pm standard deviation. Qualitative variables like gender, rise in serum creatinine $\geq 0.5 \mathrm{mg} / \mathrm{dl}$ and CIN were presented as frequency and percentage. Data was stratified for age $(30-50,51-70,>70$ years) and gender (male, female).

An approval was taken from the Ethical and research committee of Jinnah Hospital, Lahore, beforehand. 


\section{Results}

Age distribution of the patients showed; $30.32 \%(\mathrm{n}=$ 47) between $30-50$ years, $41.29 \%(n=64)$ between $51-70$ years and $28.39 \%(n=44)>70$ years of age, mean \pm SD was calculated as $58.4 \pm 13.43$ years. (Table 1).

Mean baseline creatinine level before the procedure was recorded as $0.37 \pm 0.18 \mathrm{mg} / \mathrm{dL}$, mean creatinine level post angiography was $0.88 \pm 0.22 \mathrm{mg} / \mathrm{dL}$. In the patients eventually developing CIN, mean creatinine level was found to be $0.92 \pm 2.68$ (Table 2). CIN developing in diabetics, undergoing coronary angiography was recorded in $7.10 \%(\mathrm{n}=11)$ of the total sample size, while $92.90 \%(n=144)$ had no finding of raised creatinine after angiography (Pie Chart 2).

Table 1: Age Distribution $(\mathrm{n}=155)$.

\begin{tabular}{|c||c||c|}
\hline Age (in years) & No. of Patients & $\%$ \\
\hline \hline $30-50$ & 47 & 30.32 \\
\hline \hline $51-70$ & 64 & 41.29 \\
\hline \hline$>70$ & 44 & 28.39 \\
\hline \hline Total & 155 & 100 \\
\hline
\end{tabular}

Mean \pm SD: $58.4 \pm 13.43$

Gender distribution of the patients showed $53.55 \%$ $(\mathrm{n}=83)$ male and $46.45 \%(\mathrm{n}=72)$ females (Pie Chart $1)$.

\section{GENDER DISTRIBUTION}

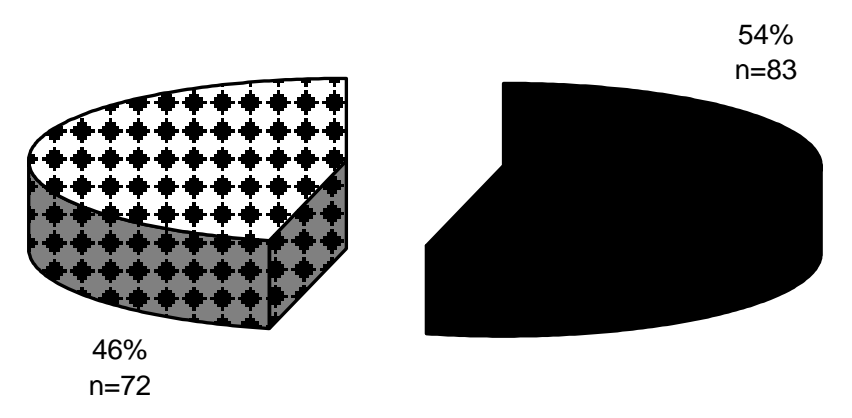

Male aFemale

Graph 1: Gender Distribution of All Cases ( $n=155)$.
Table 2: Mean Creatinine Level mg/dL ( $(\mathrm{n}=155)$.

\begin{tabular}{|l|c|}
\hline $\begin{array}{l}\text { Time of Measurement of } \\
\text { Creatinine Level }\end{array}$ & $\begin{array}{c}\text { Mean Creatinine Level } \\
(\mathrm{mg} / \mathrm{dl})\end{array}$ \\
\hline Baseline & $0.37 \pm 0.22$ \\
\hline After PCI & $0.80 \pm 0.89$ \\
\hline In cases of CIN & $0.92 \pm 2.68$ \\
\hline
\end{tabular}

Frequency of Serum Creatine $\geq 0.5 \mathrm{mg} / \mathrm{dl}$ and Contrast Induced Nephropathy in Diabetics Undergoing Angiography

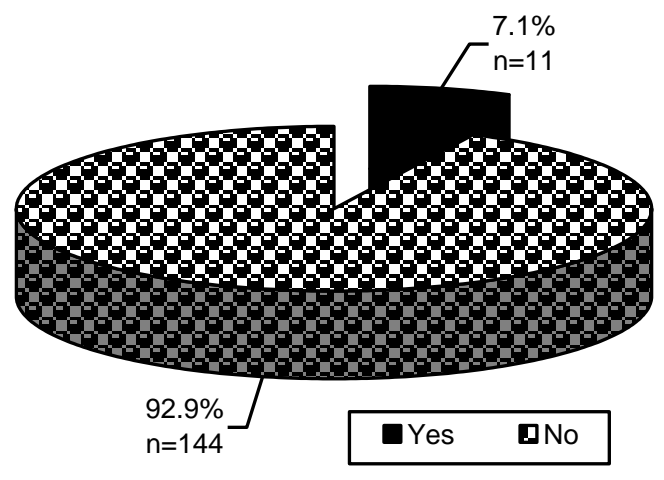

Graph 2: Frequency of Serum Creatine $\geq 0.5 \mathrm{mg} / \mathrm{dl}$ and Contrast Induced Nephropathy CIN in Diabetics Undergoing Angiography $(\mathrm{n}=155)$.

Out of 11 cases of CIN, $18.18 \%(n=2)$ were between $30-50$ years, $45.46 \%(\mathrm{n}=5)$ were between 51 70 years and $36.36 \%(n=4)$ had $>70$ years of age. (Table 3).

Out of 11 cases, $63.64 \%(n=7)$ were male and $36.36 \%(n=4)$ were females (Pie Chart 3$)$.

Table 3: Stratification for Age in Cases of CIN $(n=11)$.

\begin{tabular}{|c||c|c|}
\hline Age(in years) & No. of patients & \% \\
\hline \hline $30-50$ & 2 & 18.18 \\
\hline \hline $51-70$ & 5 & 45.46 \\
\hline \hline$>70$ & 4 & 36.36 \\
\hline \hline Total & 11 & 100 \\
\hline
\end{tabular}

\section{Discussion}

Recently CIN has caught attention due to the increasing number of cases reported and the possibility of 
lasting adverse effects following its occurrence. There has been much interest in the causative factors, pathogenesis, recognition, prevention and management on CIN in the developed countries. All this is aimed at understanding this acute injury process and to prevent the occurrence of long term adverse effects and mortality. ${ }^{10,13}$ Most clinicians have conducted observational and retrospective studies to determine the prevalence and frequency of CIN. ${ }^{12,13}$

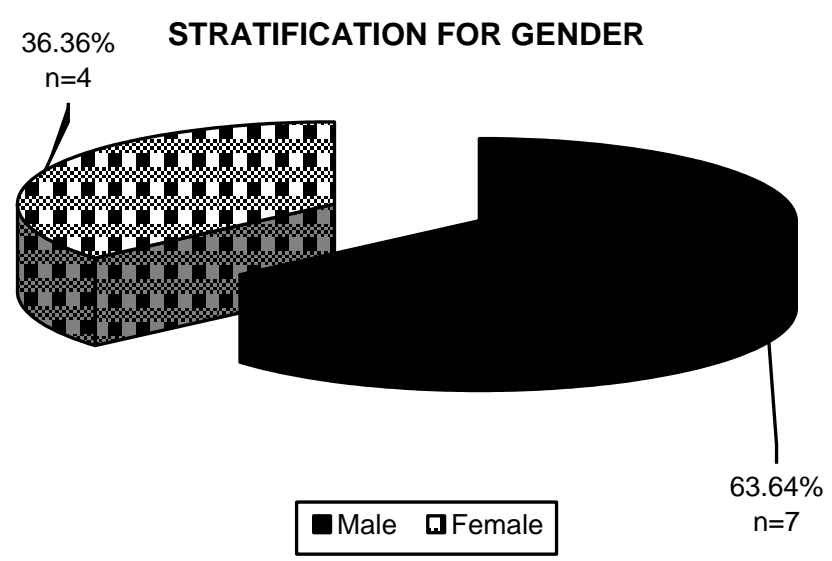

Graph 3: Stratification for Gender in Cases of CIN $(\mathrm{n}=11)$.

The reason behind this study was to determine the frequency of Contrast induced nephropathy in diabetics undergoing angiography as the literature shows that CIN is more in diabetic patients after angiography but there is also controversy in results.

In our study, out of 155 cases, mean age was calculated as $58.4 \pm 13.43$ years, $53.55 \%(\mathrm{n}=83)$ were male and $46.45 \%(n=72)$ were females. The frequency of contrast induced nephropathy in diabetics undergoing angiography was in $7.10 \%(\mathrm{n}=11)$ of the total sample studied.

Our findings are in agreement with a study that reported the frequency of CIN in diabetics was $5.2 \%$ only. ${ }^{6}$ While another study reported that frequency of CIN was $18.4 \%$ in diabetics.

Some other authors reported that, the occurrence of CIN in diabetics, previously having normal renal functions, ranges between $9-40 \%$, resulting in moderate degree of renal dysfunction. However there is a higher occurrence rate of $50-90 \%$, in diabetic patients having pre-existing renal disease. ${ }^{14,15}$ In comparison the frequency in the general population (diabetics and non-diabetics) is quite low, falling in a range of less than $2 \% .^{16}$

Diabetes is thought to be an independent risk factor for the occurrence of CIN ${ }^{17}$ However this notion has not been found to be conclusive in a lot of prospective studies. For instance, Parfreyet al, ${ }^{18}$ conducted a study focused on diabetes as a sole independent factor in the establishment of CIN and it was found that in the diabetic arm, there was no significant incidence of CIN. But still, it was concluded that the hyperglycemic patients were noted to have an increased risk of declining renal functions after exposure to contrast material. Thus it is considered prudent to count diabetes as a pre-procedural risk factor.

The findings of our study suggest that CIN remains a challenging and under-rated clinical complication occurring in diabetic patients who are undergoing coronary angiography and intervention. In order to avoid the occurrence of CIN in our setup, it is very necessary that diabetes must be taken as an independent risk factor for the development of CIN, so that pre-procedural precautions can be taken to avoid this adverse effect.

\section{Conclusion}

We concluded that the frequency of Contrast induced nephropathy is not very high in diabetic patients undergoing coronary angiography. However, it remains a clinically challenging adverse effect in diabetics undergoing coronary and these patients should be recognized and monitored carefully to avoid long-term adverse events.

\section{References}

1. Thygesen K, Alpert JS, White HD. Universal definition of myocardial infarction. J Am Coll Cardiol. 2007; 50 (22): 2173-95.

2. Zipes DP, Wellens HJ. Sudden cardiac death. Circulation, 1998; 98 (21): 2334-51.

3. Rudnick M, Feldman H. Contrast-induced nephropathy: what are the true clinical consequences? Clin J Am Soc Nephrol. 2008; 3 (1): 263-72.

4. Quintavalle C, Brenca M, De Micco F, Fiore D, Romano $\mathrm{S}$, Romano $\mathrm{M}$, et al. In vivo and in vitro assessment of pathways involved in contrast media - induced renal cells apoptosis. Cell death and disease, 2011; 2 (5): e155.

5. Rahman M, Haque H, Banerjee S, Ahsan S, Rahman M, Mahmood M, et al. Contrast induced nephropathy in diabetic and non-diabetic patients during coronary 
angiogram and angioplasty. Mymensingh Med J. 2010; 19 (3): p.372-6.

6. Worasuwannarak S, Pornratanarangsi S. Prediction of contrast induced nephropathy in diabetic patients undergoing elective cardiac catheterization or PCI: role of volume-to-creatinine clearance ratio and iodine dose-tocreatinine clearance ratio. J Med Assoc Thai. 2010; 93: S29-34.

7. Wang X-c, Fu X-h, Wang Y-b, Jia X-w, Wu W-l, Gu $\mathrm{X}-\mathrm{s}$, et al. Prediction of contrast-induced nephropathy in diabetics undergoing elective percutaneous coronary intervention: role of the ratio of contrast medium volume to estimated glomerular filtration rate. Chin Med J (Eng). 2011; 124 (6): p.892-6.

8. Sosnowski C, Janeczko - Sosnowska E, Woźniak J, Jasiński B, Dabrowski R, Sumiński A, et al. Primary coronary intervention in diabetic octogenarians with acute ST elevation myocardial infarction. Kardiologia polska. 2007; 65 (10): 1181-6.

9. Arroyo - Espliguero R, Avanzas P, Cosín - Sales J, Aldama G, Pizzi C, Kaski JC, et al. C-reactive protein elevation and disease activity in patients with coronary artery disease. Eur Heart J. 2004; 25 (3): 401-8.

10. Dangas G, Iakovou I, Nikolsky E, Aymong ED, Mintz GS, Kipshidze NN, et al. Contrast-induced nephropathy after percutaneous coronary interventions in relation to chronic kidney disease and hemodynamic variables. Am J Cardiol. 2005; 95 (1): 13-9.

11. Gruberg L, Mintz GS, Mehran R, Dangas G, Lansky AJ, Kent KM, et al. The prognostic implications of further renal function deterioration within $48 \mathrm{~h}$ of interventional coronary procedures in patients with pre-existent chronic renal insufficiency. J Am Coll Cardiol. 2000; 36 (5): 1542-8.

12. Bove T, Calabro MG, Landoni G, Aletti G, Marino G, Crescenzi G, et al. The incidence and risk of acute renal failure after cardiac surgery. J Cardiothorac Vasc Anesth. 2004; 18 (4): 442-5.

13. Best PJ, Lennon R, Ting HH, Bell MR, Rihal CS, Holmes DR, et al. The impact of renal insufficiency on clinical outcomes in patients undergoing percutaneous coronary interventions. J Am Coll Cardiol. 2002; 39 (7): 1113-9.

14. Harkonen S, Kjellstrand CM. Exacerbation of diabetic renal failure following intravenous pyelography. Am J Med. 1977; 63 (6): 939-46.

15. Barrett BJ, Parfrey PS, Vavasour HM, McDonald J, Kent G, Hefferton D, et al. Contrast nephropathy in patients with impaired renal function: high versus low osmolar media. Kidney Intl. 1992; 41 (5): 178-86.

16. Alvarez - Linera J, Benito - León J, Escribano J, Campollo J, Gesto R. Prospective evaluation of carotid artery stenosis: elliptic centric contrast-enhanced MR angiography and spiral CT angiography compared with digital subtraction angiography. Am J Neuroradiol. 2003; 24 (5): 1012-9.

17. Kolonko A, Kokot F, Wiecek A. Contrast-associated nephropathy - old clinical problem and new therapeutic perspectives. Nephrol Dial Trans. 1998; 13 (3): 803-6.

18. Schwab SJ, Hlatky MA, Pieper KS, Davidson CJ, Morris KG, Skelton TN, et al. Contrast nephrotoxicity: a randomized controlled trial of a nonionic and an ionic radiographic contrast agent. N Eng J Med. 1989; 320 (3): 149-53. 